\title{
Two-Way Denoise-and-Forward MIMO Relaying for Land Mobile Satellite Communication
}

\author{
Jiao He, Lixin Li, Jie Fan, Huisheng Zhang, Xu Li, Cai Wan \\ School of Electronics and Information \\ Northwestern Polytechnical University, Xi'an, Shaanxi 710129, China \\ E-mail:2015201314@mail.nwpu.edu.cn
}

\begin{abstract}
In this paper, a two-way denoise-and-forward (DNF) multiple-input-multiple-output (MIMO) relaying scheme for land mobile satellite (LMS) communication is proposed. We first describe the system model of two-way DNF MIMO relaying and the channel model used in LMS communication. Secondly, we use binary phase-shift keying (BPSK) to analyze the decoding bit error rate (BER) at the earth stations (ESs) and the satellite, and then obtain the end-to-end BER. Finally, we provide the simulation BER performance with BPSK modulation. The simulation results show that the two-way DNF MIMO relaying is suitable for LMS communication and the two-way MIMO with BPSK works well in LMS communication.
\end{abstract}

Keywords: Two-way relaying; multiple-input-multiple-output; land mobile satellite communication; shadowed-Rician fading; denoise-and-forward protocol.

\section{Introduction}

Land mobile satellite communication is always of great interest since it can provide various benefits like wide band transmission capability, large coverage area, and navigation assistance [1]-[3]. However, LMS communication suffers from multipath loss, shadow fading and Doppler effects, which may interrupt the communication. Also, high transmit power is demand for LMS system. Cooperative communication can provide spatial diversity to effectively resist multipath fading. And the two-way relaying can only use two time slots to complete the exchange of information, which can improve the data rate of a cooperative system compared with one-way relaying which is widely used in conventional LMS communication. MIMO technology with multiple antennas on the transceivers can promote link reliability and spectral efficiency without exact need for system bandwidth and transmit power.

There are several strategies applied for two-way relay system, such as amplified-and-forward (AF) and decode-and-forward (DF), early work of two-way AF and DF relaying can be found in [4]. A DNF protocol is proposed in [5], in which the received signal at the relay node is mapped into another symbol by applying a denoising function, and the mapped symbol can be used by each source node to decode the symbol transmitted from the other source uniquely. The DNF protocol is more efficient than DF for the relay does not need to separate message sent by source nodes. The two-way AF relaying and two-way DF relaying for satellite communication are studied in [6]-[7]. Two-way AF relaying with differential modulation for satellite communication is proposed in [8]. And [9] made a review on MIMO technologies for the satellite communications.

As summarized above, whether two-way DNF MIMO relaying is suitable for LMS communication is still an open problem. In this paper, we propose a two-way DNF MIMO relaying for LMS communication. Firstly, we describe the system model of two-way DNF MIMO relaying and the channel model used in LMS communication. Secondly, we use BPSK to analyze the decoding BER at the ESs and the satellite, and then obtain the end-to-end BER. Finally, we provide the BER performance of the two-way DNF MIMO relaying for LMS communication with BPSK modulation.

This paper is organized as follows. In Section 2, we describe the system model of two-way DNF MIMO relaying for LMS communication. In Section 3, we analyze the BER performance at the ESs and the satellite, and then obtain the end-to-end BER. In Section 4, we provide the simulation results with BSPK modulation. Finally, in Section 5, we conclude this paper.

\section{System and channel model}

We consider a two-way relaying with two ESs and a satellite, where the satellite is equipped with M antennas and the two ESs are equipped with $\mathrm{N}$ antennas, as shown in Figure 1. The two ESs communicate with each other 
simultaneously over two phases. We assume that there is no direct link between the two ESs, a half-duplex system is assumed. In this scheme, ESs don't need to know full channel state information (CSI) of both channels but only know the CSI between satellite and itself and only the satellite knows the full CSI of both channels.

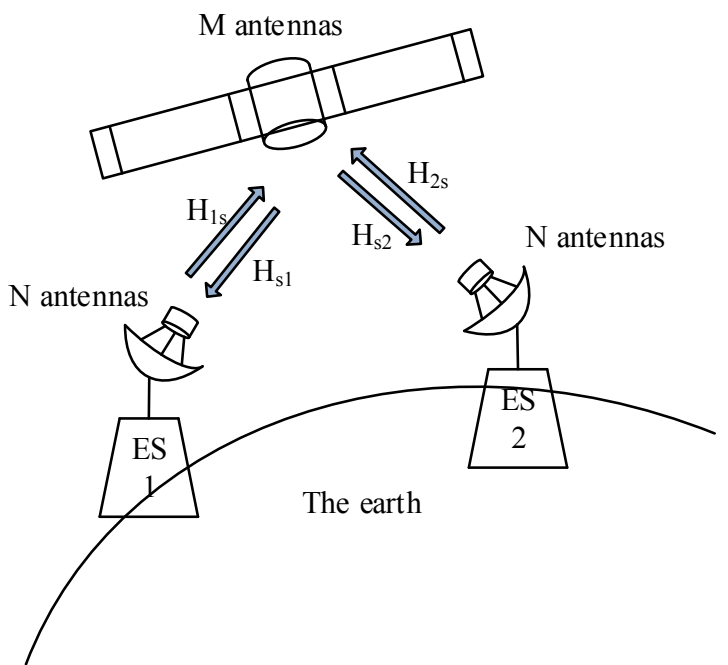

Figure 1. Two-way MIMO relaying for LMS communication

\subsection{Channel model}

The channels between the satellite and the ES are assumed to be shadowed-rician (SR) fading. The channel matrix between the satellite and the ES is given by [10]

$$
\begin{aligned}
H & =\bar{H}+\tilde{H} \\
& =A(t) \exp (j \alpha(t))+Z(t) \exp \left(j \zeta_{0}\right),
\end{aligned}
$$

Here, the entries in channel matrix $H$ are independent and identically distributed SR fading entries. $A(t)$ and $Z(t)$ denote the amplitudes of the scatter and line-of-sight (LOS) component, following Rayleigh and Nakagami distributions respectively. $\alpha(t)$ is the stationary random phase process, which satisfies the uniform distribution over $[0,2 \pi), \zeta_{0}$ represents the determined phase of the LOS component.

$$
\begin{aligned}
& p_{A}(a)=\frac{a}{b_{0}} \exp \left(\frac{-a^{2}}{2 b_{0}}\right), \quad a \geq 0 \\
& p_{Z}(z)=\frac{2 m^{m}}{\Gamma(m) \Omega^{m}} z^{2 m-1} \exp \left(\frac{-m z^{2}}{\Omega}\right), \quad z \geq 0,
\end{aligned}
$$

where $2 b_{0}=E\left[A^{2}\right]$ is the average power of the scatter component, $\Gamma(\square)$ is the gamma function, $m=\left(E\left[Z^{2}\right]\right)^{2} / \operatorname{Var}\left[Z^{2}\right] \geq 0$ is the Nakagami parameter, $\operatorname{Var}[\square]$ denotes the variance, $\Omega=E\left[Z^{2}\right]$ is the average power of the LOS component.

\subsection{System model}

In the multiple access (MAC) phase, the ES1, ES2 transmit to the satellite, the received signal at the satellite can be expressed as

$$
y_{s}=\sqrt{P_{1}} H_{1 s} M\left(x_{1}\right)+\sqrt{P_{2}} H_{2 s} M\left(x_{2}\right)+w_{s},
$$

Where $P_{1}$ and $P_{2}$ is the power of the ES1 and ES2, $H_{1 s}$ and $H_{2 s}$ denote the channel matrix from the ESs to the satellite, respectively. $M\left(x_{1}\right)$ and $M\left(x_{2}\right)$ represent the modulated signal. $w_{s}$ indicates the additive white Gaussian noise (AWGN) vector which contains complex-valued Gaussian random variables (RVs) with zero mean and variance $\sigma_{s}^{2}$.

The satellite decodes the received signal from the two ESs by using the ML decision rule as follows

$$
\hat{x}=\hat{x}_{1} \oplus \hat{x}_{2}=\underset{x_{1} \oplus x_{2}}{\arg \min _{r}}\left|y_{r}-\left(\sqrt{P_{1}} H_{1 s} M\left(x_{1}\right)+\sqrt{P_{2}} H_{2 s} M\left(x_{2}\right)\right)\right|^{2},
$$


In the broadcast $(\mathrm{BC})$ phase, the satellite broadcast the processed message to ES1 and ES2, the received signal at ES1, ES2 can be written as

$$
\begin{aligned}
& y_{1}=\sqrt{P_{s}} H_{s 1} M(\hat{x})+w_{1}, \\
& y_{2}=\sqrt{P_{s}} H_{s 2} M(\hat{x})+w_{2},
\end{aligned}
$$

Where $P_{s}$ is the transmit power of the satellite, and we have $P_{s}=P_{1}+P_{2}, H_{s 1}$ and $H_{s 2}$ denote the channel matrix from the satellite to the ESs, respectively. $w_{1}, w_{2}$ indicate the Gaussian noise with zero mean and variance $\sigma_{1}^{2}, \sigma_{2}^{2}$, respectively.

Each ES decodes the signal using the ML decision rule as follows

$$
\begin{aligned}
& \hat{\hat{x}}_{1}=\arg \min _{\hat{x}}\left|y_{1}-\sqrt{P_{s}} H_{s 1} M(\hat{x})\right|^{2}, \\
& \hat{\hat{x}}_{2}=\arg \min _{\hat{x}}\left|y_{2}-\sqrt{P_{s}} H_{s 2} M(\hat{x})\right|^{2},
\end{aligned}
$$

Each ES knows what itself transmit, therefore, the ES1 can get the message sent by ES2. Similarly, the ES2 can get the message sent by ES1.

\section{Performance analysis}

We use BPSK to analysis the end-to-end BER performance.

\subsection{Relay decoding BER}

In the multiple access (MAC) phase, the two ESs transmit message to the satellite, the satellite decoding BER is

$$
P_{s}=\frac{1}{2}\left[P\left(\hat{x}=1 \mid x_{1} \oplus x_{2}=0\right)+P\left(\hat{x}=0 \mid x_{1} \oplus x_{2}=1\right)\right]
$$

\subsection{Source decoding BER}

In the broadcast $(\mathrm{BC})$ phase, the satellite broadcast the processed message to ES1 and ES2, the BER between the message transmitted by ES1 and decoded at ES2, called $P_{2 \rightarrow 1}$, can be shown as

$$
\begin{aligned}
P_{2 \rightarrow 1} & =P\left(\hat{\hat{x}}_{1} \neq x_{1}\right)=1-\left(1-P_{s}\right)\left(1-P_{s, 1}\right)-P_{s} P_{s, 1}, \\
& =P_{s}+P_{s, 1}-2 P_{s} P_{s, 1}
\end{aligned}
$$

with $P_{s, 1}=P\left(\hat{\hat{x}}_{1} \neq \hat{x}_{1}\right)$.

Similarly,

$$
\begin{aligned}
P_{1 \rightarrow 2} & =P\left(\hat{\hat{x}}_{2} \neq x_{2}\right)=1-\left(1-P_{s}\right)\left(1-P_{s, 2}\right)-P_{s} P_{s, 2}, \\
& =P_{s}+P_{s, 2}-2 P_{s} P_{s, 2}
\end{aligned}
$$

with $P_{s, 2}=P\left(\hat{\hat{x}}_{2} \neq \hat{x}_{2}\right)$.

Finally, the total system BER is as follows

$$
\begin{aligned}
P_{\text {total }} & =\frac{1}{2}\left(P_{1 \rightarrow 2}+P_{2 \rightarrow 1}\right) \\
& =\frac{1}{2}\left[\left(P_{s}+P_{s, 1}-2 P_{s} P_{s, 1}\right)+\left(P_{s}+P_{s, 2}-2 P_{s} P_{s, 2}\right)\right]
\end{aligned},
$$

\section{Simulation results}

In this section, we provide simulation results of two-way DNF MIMO relaying for LMS communication with BPSK modulation. In this scheme, we first compare the BER performance between the MIMO and the two-way DNF MIMO relaying scheme, and then we consider how the distance between the two ESs to the satellite influence the BER. The LMS channel parameters for different shadowing scenarios are shown in Table 1. We consider infrequent light shadowing (ILS) in our simulation. And we assume that the antennas equipped in the two ESs and the satellite are same, for example, we consider $\mathrm{M}=\mathrm{N}=2$ in this scheme. $P_{1}=P_{2}$ is assumed and BER refers to the decoding error probability at ES1, that is $P_{2 \rightarrow 1}$. 
Table 1. LMS channel parameters [10]

\begin{tabular}{|c|c|c|c|}
\hline Shadowing & $b$ & $m$ & $\Omega$ \\
\hline Infrequent light shadowing (ILS) & 0.158 & 19.4 & 1.29 \\
\hline Average shadowing (AS) & 0.126 & 10.1 & 0.835 \\
\hline Frequent heavy shadowing (FHS) & 0.063 & 0.739 & 0.000897 \\
\hline
\end{tabular}

In Figure 2, we compare the simulated BER results of the MIMO and the two-way DNF MIMO relaying scheme. And in Figure 3, we compare the simulated BER results of the two-way DNF MIMO relaying with different distance between ES1 to the satellite and the ES2 to the satellite. Here, d1 denotes the distance between the ES1 and the satellite, $\mathrm{d} 2$ is the distance between the ES2 and the satellite, and $\mathrm{d}=\mathrm{d} 1+\mathrm{d} 2$ is a constant. It is obvious that the BER becomes smaller with the SNR increasing.

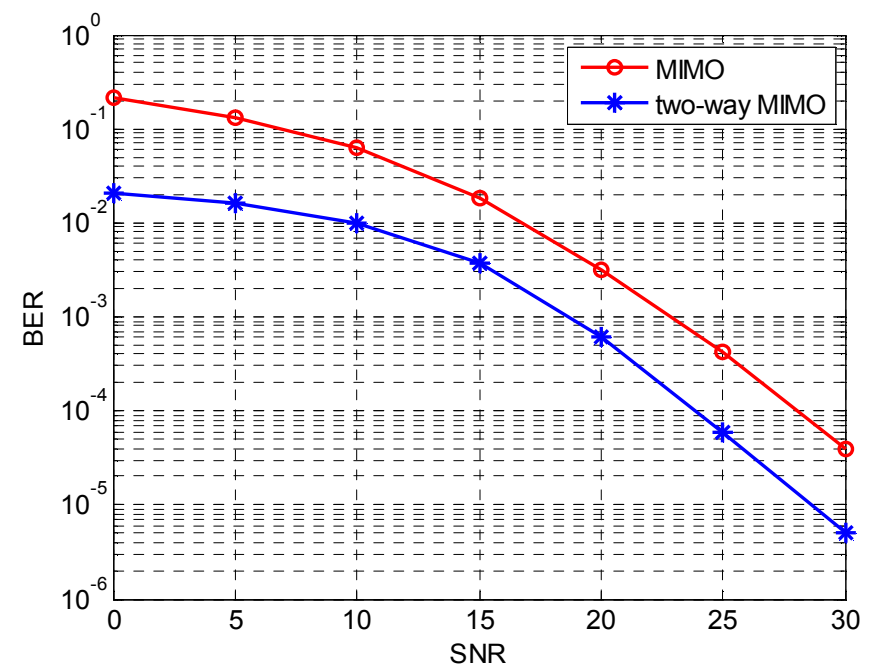

Figure 2. Simulated BER versus SNR performance between the MIMO and the two-way DNF MIMO relaying scheme, $\mathrm{d} 1: \mathrm{d} 2=1: 1$.

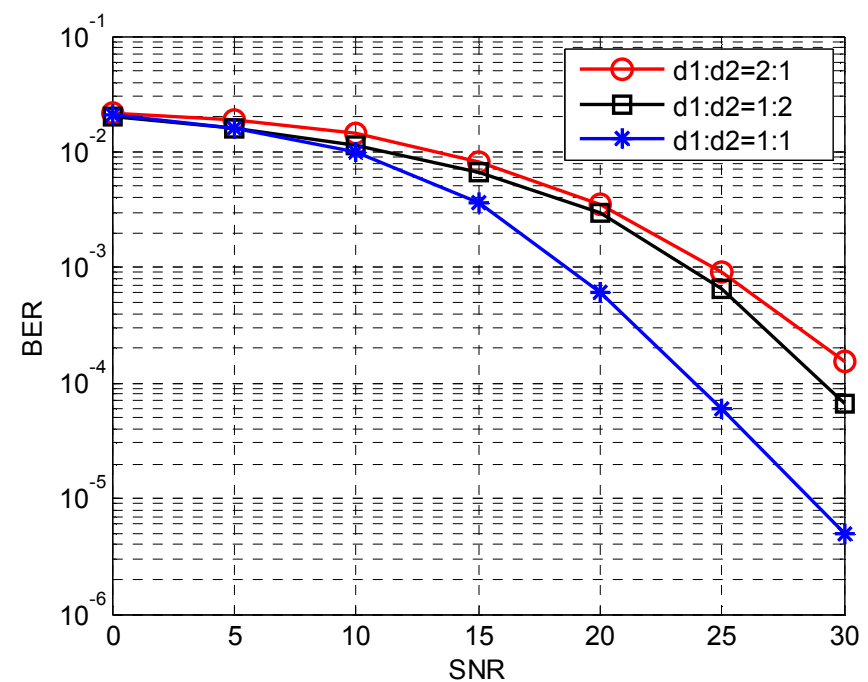

Figure 3. Simulated BER versus SNR performance of the two-way DNF MIMO relaying with different distance between ES1 to the satellite and ES2 to satellite.

From Figure 2, we observe that the BER with the two-way DNF MIMO relaying scheme is smaller than the MIMO scheme, which is because in the two-way MIMO relaying scheme, the satellite firstly decodes the received signal transmitted from the two ESs, and then broadcast the processed signal to the two ESs, thus 
reducing the BER. We can draw a conclusion that the two-way DNF relaying is suitable for LMS communication.

From Figure 3, it is apparent that the BER is different with different distance between ES1 to the satellite and ES2 to satellite. We can see that the BER is smallest when $\mathrm{d} 1: \mathrm{d} 2=1: 1$, which is due to the high channel gains of both ES to satellite channels, the BER with $\mathrm{d} 1: \mathrm{d} 2=1: 2$ is similar to $\mathrm{d} 1: \mathrm{d} 2=2: 1$, however, the BER with $\mathrm{d} 1: \mathrm{d} 2=1: 2$ is slightly smaller than that with $\mathrm{d} 1: \mathrm{d} 2=1: 2$, this is because the relaying decoding BER under the two case are same, then the end-to-end BER at the ES1 depends on the channel from the satellite to the ES1, the channel fading becomes larger with the distance increasing.

\section{Conclusion}

In this paper, we propose a two-way DNF MIMO relaying scheme for LMS communication. We analyze the decoding BER at the ESs and the satellite, and then obtain the end-to-end BER. And then we provide the simulation results of the BER performance of the two-way DNF MIMO relaying for LMS communication with BPSK modulation. The simulation results show that the two-way DNF MIMO relaying is suitable for LMS communication, the two-way MIMO with BPSK works well in LMS communication and we can get the smallest BER when the distance between ES1 to satellite and ES2 to satellite are same in the equal power allocation scenario.

\section{Acknowledgements}

This work is supported by Shaanxi Province natural science basic research plan surface project (No. 2016JM6062), by China Aerospace Science and Technology Corporation Aerospace Science and Technology Innovation Fund funded project, by the Seed Foundation of Innovation and Creation for Graduate Students in Northwestern Polytechnical University.

\section{References}

[1] T. Pratt, C. Bostian, and J. Allnutt. Satellite communications, 2nd ed. New York, NY, USA: Wiley. 2003.

[2] G. Maral, M. Bousquet, and Z. Sun. Satellite communications Systems: Systems, Techniques and Technology, 5 th ed. New York, NY, USA: Wiley. 2009.

[3] K. Y. Jo. Satellite communications Network Design and Analysis. Norwood, MA, USA: Artech House. 2011.

[4] B. Rankov and A. Wittneben. Spectral efficient protocols for half-duplex fading relay channels. IEEE Journal on Selected Areas in Communications, IEEE. 2007: 379-389.

[5] P. Popovski and H. Yomo. The anti-packets can increase the achievable throughput of a wireless multi-hop network. 2006 IEEE International Conference on Communications (ICC), 2006: 3885-3890.

[6] Ji B, Huang Y, Wang H, et al. Performance analysis of two-way relaying satellite mobile communication. 2011 6th International ICST Conference on Communications and Networking in China. IEEE. 2011: 1099-1103.

[7] M. R. Bhatnagar. Performance evaluation of decode-and-forward satellite relaying. IEEE Transactions on Vehicular Technology, 2015: 4827-4823.

[8] M. R. Bhatnagar. Making two-way satellite relaying feasible: A differential modulation based approach. IEEE Trans. Communication, 2015: 2836-2847.

[9] P. Arapoglou et al. MIMO over satellite: A review. IEEE Communications Surveys \& Tutorials, 2011: $27-51$.

[10] A. Abdi, W. Lau, M.-S. Alouini, and M. Kaveh. A new simple model for land mobile satellite channels: First and second order statistics. IEEE Transactions on Wireless Communications, 2003: 519-528. 